\title{
Influência da reabilitação vestibular em indivíduos com desequilíbrio postural
}

\author{
The influence of the vestibular rehabilitation in patients with postural imbalance
}

Adriana Mazzucato', Ana Paula Oliveira Borges ${ }^{2}$

\begin{abstract}
RESUMO
Introdução. O sistema vestibular é uma ferramenta no controle da postura e das funções sensório-motoras. Os sinais e sintomas mais comuns na disfunção vestibular são a tontura rotatória ou a vertigem, perda de audição ou zumbido, presença de nistagmo e alteração da postura e do equilíbrio. Este trabalho teve como objetivo avaliar os resultados da reabilitação vestibular em pacientes com desequilíbrio postural. Método. Participaram deste estudo quatro pacientes com idade média de $47 \pm 6$ anos, apresentando diagnóstico de disfunção vestibular periférica que foram avaliados e tratados (duas vezes por semana com duração de uma hora) na clínica escola de fisioterapia da UNIFRAN durante sete meses. Para avaliação do equilíbrio estático e dinâmico foram aplicadas as escala de equilíbrio de Berg, de Tinetti e o Timed Up and Go. Resultados. Todos os pacientes apresentaram aumento no equilíbrio estático e dinâmico de acordo com as médias das escalas de Berg (52), Tinetti (24,75) e timed up and go $(11,35)$ quando se comparadas com as médias da avaliação inicial da escala de Berg (45), Tinetti $(21,25)$ e timed up and go $(12,61)$. Conclusão. Os dados sugerem que a reabilitação vestibular foi efetiva para aumentar o equilíbrio estático e dinâmico nesses pacientes.

Unitermos. Doença Vestibular, Equilíbrio Musculosquelético, Reabilitação.

Citação. Mazzucato A, Borges APO. Influência da reabilitação vestibular em indivíduos com desequilíbrio postural
\end{abstract}

Trabalho realizado no Setor de Neurologia da Universidade de Franca, Franca-SP, Brasil.

1. Pós-Graduanda do Curso de Fisioterapia Neurológica da Universidade de Franca - UNIFRAN, Franca-SP, Brasil.

2. Fisioterapeuta, Mestre, professora de Fisioterapia da UNIFRAN e UNIARAXÁ, Franca-SP, Brasil.

\section{SUMMARY}

Introduction. The vestibular system is a toll to posture control and to sensory motor functions. The signs and symptoms more commons are vertigo rotation and dizziness, loss of the audition or buzzing, presence of nistagmus, and posture and balance alteration. This study had the aim to assess the vestibular rehabilitation results in postural imbalance patients. Method. Four patients, mean age $47 \pm 6$ years with peripheral vestibular dysfunction diagnosis that were evaluated and treated (twice a week, one-hour session) at Physical Therapy Clinic School of UNIFRAN, for seven months. The static and dynamic balance was assessed with Berg Balance Scale, Performance mobility Assessment POMA (Tinetti), and Timed Up and Go test. Results. All patients had increased static and dynamic balance in the Berg Balance Scale (52), Tinetti (24.75), and Timed Up and Go test (11.35) when compared with the baseline score: Berg Balance Scale (45), Tinetti (21.25), and Timed Up and Go test (12.61). Conclusion. The data suggest that the vestibular rehabilitation was effective to improve static and dynamic balance in those patients.

Keyword. Vestibular Disease, Musculoskeletical Equilibrium, Rehabilitation.

Citation. Mazzucato A, Borges APO. The influence of the vestibular rehabilitation in patients with postural imbalance.

Endereço para correspondência: Ana Paula O Borges Clínica de Fisioterapia - UNIFRAN Av. Armando S Oliveira, 201 CEP 14.404-600, Caixa Postal 082, Franca-SP, Brasil e-mail: anaproliveira@unifran.br 


\section{INTRODUÇÃO}

Disfunção vestibular periférica envolve os órgãos terminais vestibulares e/ou os nervos vestibulares $^{1}$. O sintoma característico é a tontura rotatória ou a vertigem. A vertigem é um sintoma muito prevalente, encontrado em $10 \%$ da população mundial e em $85 \%$ dos casos ela é decorrente de disfunção vestibular $^{2}$. Outros sinais e sintomas seriam a perda de audição ou zumbido ${ }^{3}$ presença de nistagmo ${ }^{4}$, náuseas, vômitos, sudorese, palidez e desequilíbrio postural ${ }^{5}$.

Uma das funções mais importantes do sistema do controle postural humano é a do equilíbrio do corpo sobre a pequena base de apoio sobre os pés. O sistema vestibular, como um sensor da gravidade, é uma das ferramentas mais importantes do sistema nervoso no controle da postura. Ele possui funções sensoriais e motoras. Na sua função sensorial, o sistema vestibular fornece ao Sistema Nervoso Central (SNC) informações sobre a posição e o movimento da cabeça e a direção da gravidade. O SNC usa essas informações, combinadas com as fornecidas por outros sistemas (visual e somatossensorial), para construir uma imagem da posição e do movimento do corpo todo e do ambiente que o cerca. $\mathrm{Na}$ sua função motora, o SNC utiliza as vias motoras descendentes, que recebem informações vestibulares e de outros tipos, para controlar as posições estáticas da cabeça e do corpo e também para coordenar os movimentos posturais.

Estudos têm também mostrado que pessoas com disfunção vestibular andam mais devagar com a base de suporte alargada, viram em bloco, e são receosos em relação a movimentos repentinos ${ }^{7,8}$. Além disso, habilidades na posição de equilíbrio têm sido mostrados estar prejudicados e a velocidade da marcha reduzida em pacientes com disfunção vestibu$\operatorname{lar}^{9-11}$.

A reabilitação vestibular tem se tornado extremamente útil em pacientes com vertigem, desequilíbrio e instabilidade na marcha. Em vários estudos, são evidenciados a eficácia da fisioterapia vestibular em pacientes com desordens vestibulares periféricas ${ }^{11-13}$ e com disfunção vestibular central ${ }^{14,15}$. Contudo, Shepard et al. ${ }^{16}$ demonstraram que o local da lesão (periférico, central, misto) não influencia no resultado final como medida de índices globais de sintomas e incapacidades vestibulares.

Estudos têm se direcionado a demonstrar a influencia da reabilitação vestibular para esses pacientes e o desequilíbrio postural e suas repercussões devem ser melhor investigadas. Este trabalho teve como objetivo avaliar o equilíbrio estático e dinâmico em pacientes com desequilíbrio postural decorrentes de disfunção vestibular periférica.

\section{MÉTODO}

\section{Amostra}

Participaram deste estudo quatro pacientes (três mulheres e um homem), com idade média de $47 \pm 6$ anos, apresentando diagnóstico clínico de disfunção vestibular periférica, através de encaminhamento médico, com sinais e sintomas de tontura rotatória ou vertigem, zumbido, déficit de equilíbrio, náuseas e oscilopsias.

O estudo foi aprovado pelo comitê de ética desta instituição através do protocolo de n 133/06 e os pacientes foram informados sobre os procedimentos e assinaram um termo de consentimento livre e esclarecido.

\section{Procedimentos}

Os pacientes foram avaliados e tratados (duas vezes por semana com duração de uma hora a sessão) na clínica escola de fisioterapia da Universidade de Franca durante sete meses.

Os exercícios habituais foram baseados na exposição de repetição para provocar estímulos que resultem na redução dos sintomas. $\mathrm{O}$ tratamento proposto para esses indivíduos era a realização de atividades que enfocassem o equilibro, a marcha, mudanças de posturas e treinos adaptativos para vertigem. As manobras terapêuticas utilizadas foram à manobra liberatória de Semont, a manobra de reposicionamento dos debris de estatocônios de Epley, os exercícios de Brandt e daroff e a manobra de Lempert conforme demonstrada por estudo literário $^{8}$. As manobras terapêuticas variaram de acordo com o canal semicircular acometido. Foi realizada, ainda, treino de equilíbrio em diferentes posicionamentos e ambientes e treino de marcha (com obstáculos e superfícies irregulares).

\section{Avaliação}

Para a avaliação dos benefícios da reabilitação, foram utilizados na avaliação as escalas de equilíbrio de Berg ${ }^{10,11}$, avaliação da mobilidade orientada para o desempenho POMA I - Tinetti ${ }^{12}$ e o teste Timed Up and Go (levante-se e ande cronometrado) $)^{13,14}$. 
O primeiro consiste em 14 tarefas multifuncionais comumente realizadas na vida diária, tais como mudanças da posição sentada para a posição em pé e vice-versa, variações na posição em pé (olhos fechados, pés juntos, com os braços estendidos à frente, recolhendo um objeto do chão, girando e em pé sobre um pé) e colocando um pé sobre um banquinho. É usada uma escala ordinal de cinco pontos, com pontuações variando de zero a quatro pontos, no qual o número quatro é usado para identificar que o paciente consegue realizar a tarefa de maneira independente e de acordo com os critérios de tempo e distância; e o número zero é utilizado quando o paciente é incapaz de realizar a tarefa. A pontuação máxima é de 56 pontos $^{17,18}$.

O segundo inclui itens de equilíbrio estático e dinâmico tais como equilíbrio sentado, levantar e sentar em uma cadeira, equilíbrio em pé (olhos abertos, fechados, suportando uma perturbação, apoio sobre uma perna, apoio com os pés alinhados, estendendo os braços à frente, curvando-se para frente e girar 360 ), avaliação da marcha (início, desvio do trajeto, subida de degraus, giro e tempo de marcha). Alguns itens são pontuados numa escala de zero a dois, e outros são cronometrados. A pontuação total é de $28^{19,20}$.

No terceiro, o paciente senta-se confortavelmente em uma cadeira firme com os braços e as costas apoiados na cadeira; é então instruído a levantar-se (é cronometrado quando o paciente recebe o comando "vá"), ficar parado momentaneamente e depois caminhar um metro e meio em direção a uma parede usando a velocidade de marcha normal, girar sem tocar na parede, retornar para a cadeira, girar e sentar-se. As pesquisas indicam que a maioria dos adultos pode completar o teste em dez segundos.
As pontuações de 11 a 20 segundos são consideradas dentro dos limites normais para idosos frágeis ou indivíduos com incapacidade; pontuações acima de 20 segundos são indicativas de mobilidade funcional comprometida ${ }^{13,14}$.

\section{Análise estatística}

Após a avaliação inicial e final, os resultados foram comparados entre si através do teste $t$ de Student.

\section{RESULTADOS}

Todos os indivíduos apresentaram uma melhora significativa do equilíbrio estático e dinâmico quando comparadas às avaliações finais com as iniciais. Os valores individuais podem ser observados nas Figuras 1, 2 e 3 que contêm dados referentes aos valores obtidos (antes e após a reabilitação vestibular) através da escala de Berg, escala de Tinetti e Timed up and go, respectivamente.

A escala de Berg foi sensível às mudanças de postura exigidas nos itens avaliados e as atividades de rotação. A escala de Tinetti evidenciou melhora no desempenho da marcha e melhor habilidade no equilíbrio dinâmico e o teste Timed up and go reproduziu os melhores resultados nas escalas de Berg e Tinetti através do menor tempo de execução da tarefa feito por todos os indivíduos.

\section{Discussão}

Todos os sujeitos participantes deste estudo apresentaram diminuição no equilíbrioestático e dinâmico na avaliação inicial, evidenciando a diminuição nas atividades que requerem mobilidade rápida, equilíbrio dinâmico e marcha conforme as médias apresentadas na escalas avaliadas.

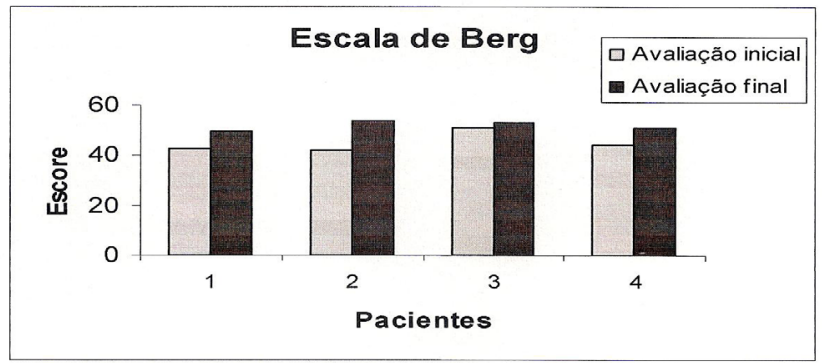

Figura 1. Representação gráfica dos resultados individuais da aplicação da Escala de Berg. 


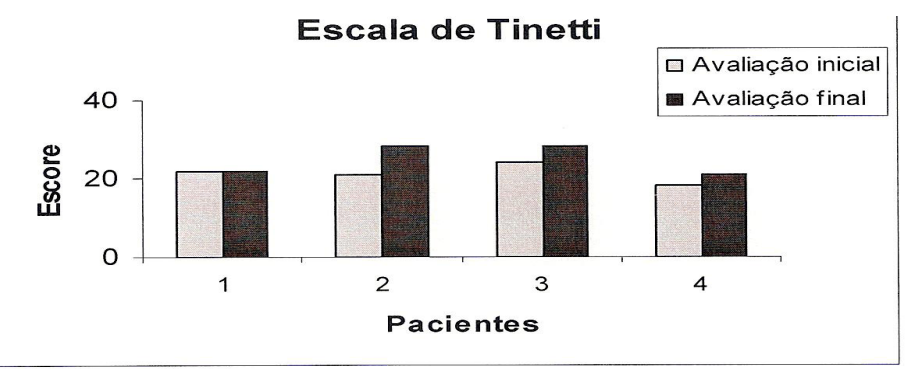

Figura 2. Representação gráfica dos resultados individuais da aplicação da Escala de Tinetti.

A reabilitação vestibular facilita adaptação para substituir ou alterar a função vestibular ${ }^{15}$; melhora a estabilidade da marcha (incluindo controle cinético em resposta a perturbações mal antecipadas); melhora dos sintomas desencadeantes pelo movimento; corrige dependências exageradas (seleção sensorial inapropriada) do sistema visual e somatossensorial; facilita o retorno normal das atividades de vida diária e, melhora ou restaura a condição neuromuscular. O elemento chave para o sucesso na reabilitação vestibular está no procedimento ensinado ao paciente para identificar e utilizar mais corretamente a informação sensorial (espacial) disponível durante os movimentos ativos e passivos do corpo.

A postura é controlada pelo sistema visual, somatossensorial e vestibular, que são integrados pelo sistema nervoso central. $\mathrm{O}$ objetivo do controle postural é manter a posição do corpo estável e de maneira orientada ${ }^{16}$.

A reabilitação vestibular representa uma terapia fisiológica, sem reações colaterais e extremamente eficientes. Muitos pacientes que apresentam limitações severas em suas atividades devido à sintomatologia intensa respondem muito bem à terapia com exercícios.
As razões da melhora da reabilitação labiríntica são decorrentes das adaptações neurais multifatoriais, substituições sensoriais, recuperação funcional dos reflexos vestíbulo-ocular e vestíbulo- espinhal, condicionamento global e alteração do estilo de vida.

A reorganização sensoriomotora, que conduz uma grande estabilidade postural, está relacionada à extensa quantidade de instabilidade postural evidenciado no treinamento. Logo, para facilitar a reorganização e o recrutamento da capacidade de controle, devemos aumentar a exposição do paciente com disfunção vestibular em posições instáveis do corpo $^{17}$.

Os resultados apresentados neste estudo após a reabilitação vestibular foram benéficos já que os pacientes melhoraram o equilíbrio estático e dinâmico de acordo com as médias apresentadas na escalas de Berg (52), Tinetti $(24,75)$ e Timed up and go $(11,35)$. Essa melhora desencadeou a diminuição dos sintomas de vertigem além de aumentar a independência nas atividades de vida diária e diminuir o risco de quedas, conforme relato dos pacientes. Esses resultados corroboram com os resultados realizados por outros autores ${ }^{18-21}$.

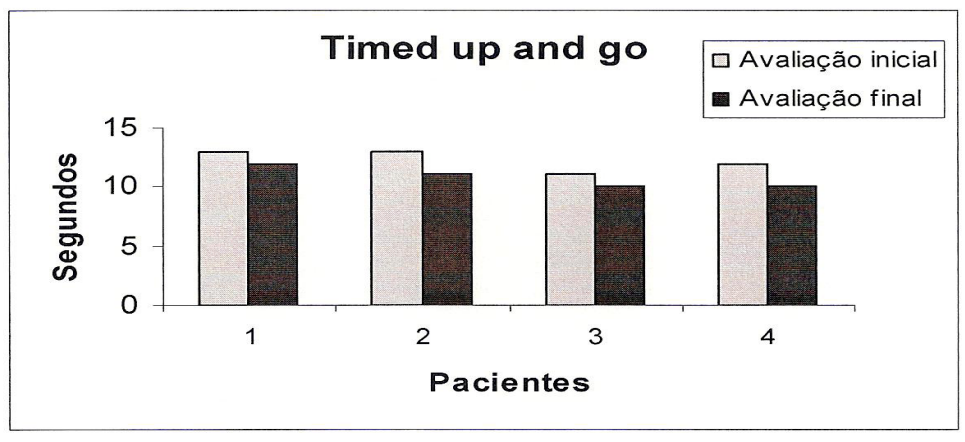

Figura 3. Representação gráfica dos resultados individuais da aplicação do teste Timed up and go. 
Macias et al. ${ }^{22}$ estudaram 70 pacientes com idade mínima de 50 anos com diagnóstico de vertigem de origem central e periférica, infarto cerebelar, desequilíbrio na marcha e disfunção vestibular periférica. Foi avaliado o risco de queda através da escala de Berg.

A reabilitação vestibular resultou numa melhora estatisticamente significante de acordo com a escala utilizada (antes da reabilitação: 36,8 e após da reabilitação: 46,4).

Portanto, o tratamento resultou numa significante redução no risco de queda, sugerindo que a reabilitação vestibular tem um impacto positivo em prevenir e evitar distúrbios de equilíbrio e na marcha em idosos.

Um programa básico de habituação vestibular com exercícios de movimentação de cabeça reduziu os sintomas de vertigem (intensidade e freqüência) e aumentou a independência nas atividades de vida diária ${ }^{23}$.

Outras pesquisas evidenciaram que a reabilitação vestibular tornou-se muito utilizada para pacientes com vertigem, desequilíbrio e com instabilidade na marcha e, que foi efetiva para a diminuição destes sintomas ${ }^{24}$. Estes trabalhos embasam os resultados deste estudo, uma vez que a reabilitação vestibular trouxe benefícios não somente de ordem física, mas também funcional $\mathrm{e}$ emocional aos pacientes com disfunção vestibular.

Os benefícios dos programas de reabilitação vestibular estão se tornando extremamente aceitáveis, envolvendo tipicamente três caminhos: exercícios de habituação designados para facilitar a compensação do sistema nervoso central pela extinção patológica da resposta do movimento da cabeça; exercícios de controle postural, e várias atividades condicionantes $^{7}$. A função primordial do terapeuta no tratamento de pacientes com déficit de equilíbrio é educá-los quanto a sua doença. Existe com freqüência uma considerável falta de informação que deve ser esclarecido. Pacientes que são bem informados a respeito da origem da disfunção vestibular entenderão a razão física para a reabilitação vestibular e por isso reconhecerão que essas medidas são simplesmente um tratamento prático para a cura.

Estudos têm mostrado que muitos pacientes tratados com reabilitação vestibular mostram ao menos uma melhora moderada nos seus sintomas, e poucos pacientes mostram uma dramática ou completa melhora na resolução dos sintomas. Estes estudos têm utilizado diferentes medidas pra quantificar/qualificar os sintomas e funções para muitos pacientes, mas, não para todos os pacientes com disfunção vestibular ${ }^{25-27}$.

Por fim, esse presente trabalho corrobora com estes últimos estudos, uma vez que nem todos os participantes desse trabalho conseguiram apresentar $100 \%$ de melhora na resolução dos sintomas e, mesmo assim, os resultados mostram que a reabilitação vestibular foi eficaz para a melhora do equilíbrio e, conseqüentemente, isso refletiu na diminuição dos outros sinais e sintomas.

\section{CONCLUSÃO}

A proposta deste estudo foi avaliar os resultados da reabilitação vestibular em pacientes com desequilíbrio postural e os dados apresentados evidenciaram que a reabilitação vestibular foi efetiva para melhorar o equilíbrio estático e dinâmico apresentados por esses pacientes.

\section{REFERÊNCIAS BIBLIOGRÁFICAS}

1.Fetter M. Disfunções do sistema vestibular. In: Herdman SJ. Reabilitação vestibular. 2. ed. Barueri: Manole, 2002, p. 91-101. 2.Menon AD, Campos CAH, Sakano E, Weckx LLM, Ganança MM. Fórum sobre vertigem. Rev Bras Otorrinolaringol 2003;69(supp1):106-39.

3.Goebel JA. The ten-minute examination of the dizzy patient. Sem Neurol 2001;21:391-8.

4.Baloh RW. Clinical practice: Vestibular neuritis. New Eng J Med 2003:384:1027-32.

5.Herdman SJ. Reabilitação vestibular. 2. ed. Barueri: Manole, 2002, 621p.

6.Buzatti DRP, Albertin C, Carmona ST, Oliveira AEAL, Byrro C, Roberto L. Reabilitação Vestibular. Fisioter Bras 2007;8:4752.

7.Herdman SJ, Clendaniel RA, Mattox DE, Holliday MJ, Niparko JK. Vestibular adaptation exercises and recovery: acute stage after acoustic neuroma resection. Otolaryngol Head Neck Surg 1995;113:77-87.

8.Shepard NT, Telian SA. Programmatic vestibular rehabilitation. Otolaryngol Head Neck Surg 1995;112:173-82.

9.Shepard NT, Telian SA, Smith-Wheelock M, Raj A. Vestibular and balance rehabilitation therapy. Annals Otol Rhinol Laryngol 1993;102:198-205.

10.Miyamoto ST, Lombardi Jr I, Berg KO, Ramos LR, Natour J. Brazilian version of the Berg balance scale. Braz J Med Biol Res 2004;37:1411-21.

11.Berg KO, Maki BE, Williams JI, Holliday PJ, Wood-Dauphinee SL. Clinical and laboratory measures of postural balance in an elderly population. Arch Phys Med Rehabil 1999;273:107380.

12.Tinetti M. Performance-oriented assessment of mobility problems in elderly patients. J Am Geriatr Soc 1986;34:119-26.

13.Tinetti M, Williams TF, Mayewski R. Fall risk index for elderly patients based on number of chronic disabilities. Am J Med 1986;80:429-34.

14.Mathias S, Nayak US, Isaacs B. Balance in elderly patients: 
The "Get-up and go" test. Arc Phys Med Rehab 1986;67:387-9. 15.Black FO, Pesznecker SC. Vestibular adaptation and rehabilitation. Cur Opin Otolaryngol Head Neck Surg 2003;11:355-60. 16.Rama-Lopez J, Pérez N, Vila EM. Dynamic posture assessment in patients with peripheral vestibulopathy. Act Otolaryngol 2004;124:700-5.

17.Bronstein AM.Vertigo Itsmultisensory syndromes. Brain2000;123:849-51.

18.Ribeiro ASB, Pereira JS. Melhora do equilíbrio e redução da possibilidade de quedas em idosas após os exercícios de Cawthorne e Cooksey. Rev Bras Otorrinolaringol 2005;71:38-46.

19.Badke MB, Shea TA, Miedaner JA, Grove CR. Outcomes after rehabilitation for adults with balance dysfunction. Arc Phys Med Rehab 2004;85:227-33.

20.Cohen HS, Kimball KT. Decreased ataxia and improved balance after vestibular rehabilitation. Otolaryngol Head Neck Surg 2004;130:418-25.
21.Murray K, Carroll S, Hill K. Relationship between change in balance and self-reported handicap after vestibular rehabilitation therapy. Physiother Res Inter 2001;6:251-63.

22.Macias JD, Massingale, S, Gerkin RD. Efficacy of vestibular rehabilitation therapy in reducing falls. Otolaryngol Head Neck Surg 2005; 133:323-5.

23. Cohen HS, KimbalL KT. increased independence and decreased vertigo after vestibular rehabilitation. Otolaryngol Head Neck Surg 2003;128:60-70.

24.Cass SP, Borello-France D, Furman JM. Functional outcome of vestibular rehabilitation in patients with abnormal sensoryorganization testing. Am J Otol 1996;17:581-94.

25.Telian SA, Shepard NT, Smith-Wheelock M, Kemink JL. Habituation therapy for chronic vestibular dysfunction: preliminary results. Otolaryngol Head Neck Surg 1990;103: 89-95.

26.Telian SA, Shepard NT, Smith-Wheelock M, Hoberg M. Bilateral vestibular paresis: diagnosis and treatment. Otolaryngol Head Neck Surg 1991;104:67-71.

27.Keim RJ, Cook M, Martini D. Balance rehabilitation therapy. Laryngos 1992;102:1302-7. 